\title{
Hierarchical Successive Stream Selection for Heterogeneous Network Interference
}

\author{
Esra Aycan*, Berna Özbek* and Didier Le Ruyet ${ }^{\dagger}$ \\ *Electrical and Electronics Engineering Department \\ İzmir Institute of Technology, İzmir, Turkey \\ Email: esraaycan, bernaozbek@iyte.edu.tr \\ ${ }^{\dagger}$ CEDRIC/LAETITIA, CNAM, Paris, France \\ Email: leruyet@cnam.fr
}

\begin{abstract}
This paper presents a hierarchical stream selection approach to deal with the interference in a heterogeneous network where different cell types are coexisting with each other to increase the sum capacity. Due to the variety of the transmit powers between the macro and small cells, interference levels are different. The proposed solution hierarchically selects the strongest streams of each cell with a contribution to the sum rate, while constructing the streams via singular value decomposition (SVD). In order to reduce the interference, the channel matrices of the remaining streams are projected orthogonally to the virtual transmit channel and virtual receive channel of the selected stream. The performance evaluations are obtained by considering different locations of small cells with respect to the macro cell. It is shown that the proposed method can dynamically select more streams in heterogeneous networks and achieve higher data rates compared to the existing algorithms.
\end{abstract}

\section{INTRODUCTION}

As the demand of higher data rates and the quality of service are increasing in wireless communication, innovative approaches and solutions are seeking for next generation systems. Novel wireless system architectures and models are required in order to provide the necessary capacities to support high data rate services. Heterogeneous networks provide a large number of new node deployment of smaller cells with different power levels (micro, pico or femto cells) in the coverage of the conventional macro cell using the same spectrum. An illustration of a heterogeneous cellular network composed of pico cells and macro cell is illustrated in Figure 1.

Although this overlaying cellular network provides coverage extension, interference management is required for the performance enhancement. There are different kinds of interference management approaches which are investigated in a heterogeneous environment. These approaches are classified into three major categories such as interference cancellation through receiver processing, interference randomization via frequency hopping, and interference avoidance achieved by restrictions imposed in resource usage in terms of resource partitioning and power allocation. The main point of the interference management is to achieve the coordination among small cells with a well-designed beamforming and space time coding techniques.

Another effective interference mitigation technique referred as interference alignment (IA) has been recently investigated for wireless networks. This scheme is introduced as a linear precoding technique that aligns interfering signals in time,

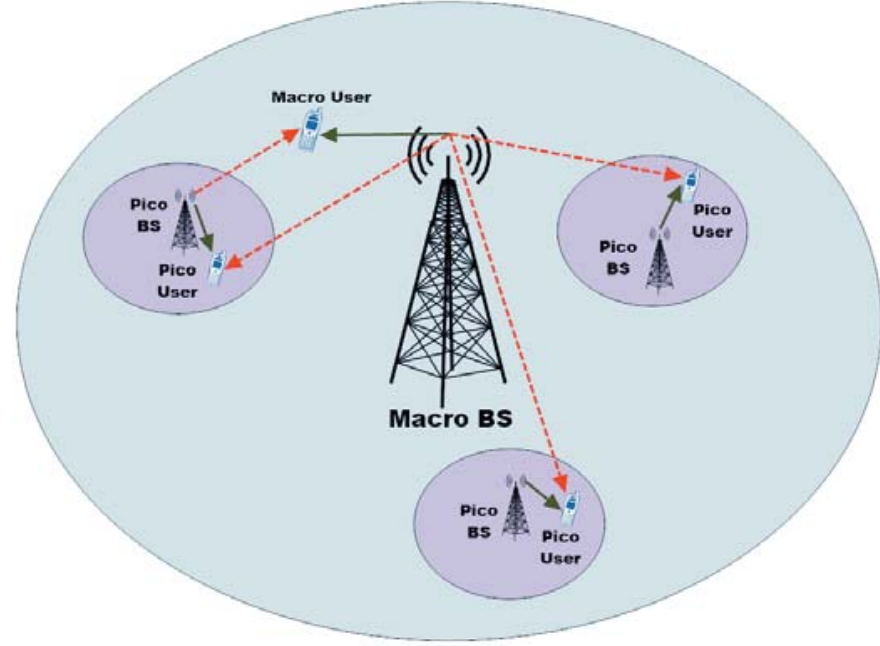

Fig. 1. Heterogeneous Network Architecture. Solid red lines show useful signals. Green dashed lines show interference signals.

frequency, or space. The IA studies are started with focusing on $\mathrm{K}$ pair interference channels where each transmitter has a message for only one of the receivers. In the study of [1], performance results of the IA method show that the throughput capacity of the network linearly grows when the size of the network increases. It is shown that all interference can be concentrated on one half of the signal space at each receiver, leaving the other half available to the desired signal and free of interference. Further studies on $\mathrm{K}$ pair interference channels can be found in [2], [3].

The first study on implementing IA methods to cellular networks is given in [4]. Authors apply sub-space IA approach to cellular systems in order to increase the throughput of the network considering the cell-edge area. In the study [5], Maximum Signal to Interference plus Noise Ratio (maxSINR) algorithm given in [6] is extended for the cellular networks. The authors in the study of [7] present a stream selection procedure by selecting the least interfering streams successively to be in the null space of the previously selected ones. In order to relax the constraint of the feasibility bounds in the IA approach, the streams are selected by allowing some interference that can be ignored as long as this does not affect the sum rate of the system. 
On the other hand there are few studies on IA for heterogeneous networks to handle the problems caused by the coexistence of macro and small cells. In the study of [8], a spectral transmission scheme for femto cell networks, which includes an adaptive subband partition method and an adaptive IA transceiver is introduced. Another IA approach on femtocellular networks is given in [9] where the uplink interference is tried to be aligned caused by the macro cell users to the closest femtocell by satisfying the required quality of service (QoS). In the study of [10] beamforming matrices are sequentially determined for small cells and macro cells in order to mitigate interference in the heterogeneous networks. Authors design the IA method based on based on the assumption that the number of antennas in macro base stations (BSs) is higher than the number of antennas in pico BSs. The transmit beamforming matrices are successively constructed according to the ascending order of the number of transmit antennas in order to align the interference vectors in a small dimensional space.

In this paper heterogeneous network interference is handled by an orthogonalization procedure on the hierarchically selected streams. The streams are initially selected from the user of the pico cell, continuing with the strongest streams among the remaining streams that positively contribute to the sum rate. Orthogonalization procedure is carried out after selecting each stream. The emerging stream combinations are compared with each other and the combination leading to the greatest sum rate is chosen. The main aim is to increase the overall capacity of the system designing the precoding and decoding matrices while mitigating the interference.

The sections are organized as follows. Section II describes the system model. In Section III the proposed algorithm is presented. Section IV gives the simulation results and finally the paper is concluded in Section V.

\section{SySTEM MODEL}

In this study, a K-pair heterogeneous network is considered as a system model composed of pico cells and macro cells with $N_{T_{k}}$ transmitter antennas and $N_{R_{k}}$ receiver antennas as seen in Figure 2. It is assumed that perfect channel state information (CSI) is available at all transmitters and receivers.

The notations used in this paper are defined as: $\operatorname{rank}(\mathbf{A})$ is the rank of the matrix $\mathbf{A},(\mathbf{A})^{H}$ represent the transpose conjugate of the matrix $\mathbf{A}$ and $|\mathbf{A}|$ represents the determinant of square matrix A. Capital Greek letters such as $\Omega$ denote sets and $|\Omega|$ denotes the number of elements of set $\Omega$.

The transmission channel matrix is $\mathbf{H}_{k k}$ with dimension $N_{R_{k}} \times N_{T_{k}}$. Each element of the channel matrix includes channel effects such as path loss and multipath fading. The interference channel from transmitter $j$ to user $k$ is given as $\mathbf{H}_{k j}$. The received signal at user $k$ as

$$
y_{k}=\mathbf{H}_{k k} \mathbf{T}_{k} \mathbf{x}_{k}+\sum_{j=1, j \neq k}^{K} \mathbf{H}_{k j} \mathbf{T}_{j} \mathbf{x}_{j}+\mathbf{n}_{k}
$$

where $\mathbf{n}_{k}$ is the additive white Gaussian noise vector with a size of $N_{R_{k}} \times 1$ with zero mean and variance $\sigma^{2}$ at each receiver $k$. $\mathbf{T}_{k}$ is the beamforming matrix of the $k^{t h}$ transmitter

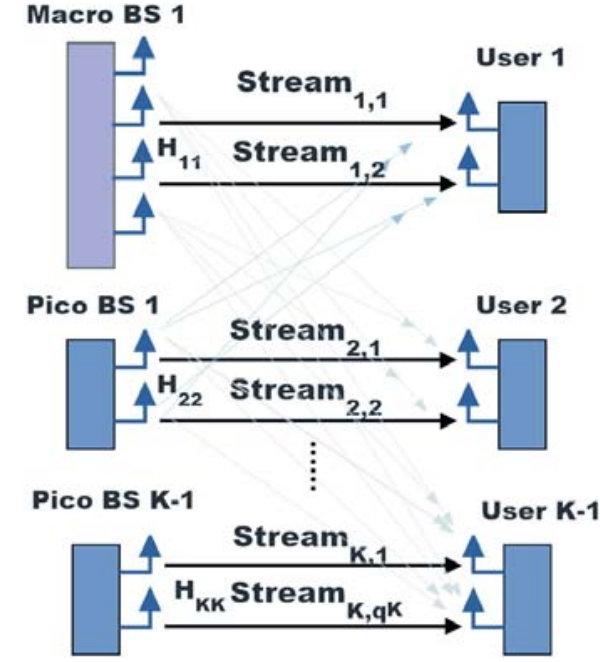

Fig. 2. System Model for MIMO Heterogeneous Network

with a size of $N_{T_{k}} \times q_{k}$, and transmitter $k$ can transmit $q_{k}$ independent streams at most $\min \left(N_{R_{k}}, N_{T_{k}}\right) . \mathbf{x}_{k}$ is the transmit data symbol vector with the size of $q_{k} \times 1$ and denoted as $\mathbf{x}_{k}=\left[x_{k, 1} \ldots x_{k, q_{k}}\right]$.

Desired signals are decoded by multiplying them by the receive beamforming vector, $\mathbf{D}_{k}$ with a size of $q_{k} \times N_{R_{k}}$. The obtained decoded data symbols can be shown as

$$
\hat{y}_{k}=\mathbf{D}_{k} \mathbf{H}_{k k} \mathbf{T}_{k} \mathbf{x}_{k}+\sum_{j=1, j \neq k}^{K} \mathbf{D}_{k} \mathbf{H}_{k j} \mathbf{T}_{j} \mathbf{x}_{j}+\mathbf{D}_{k} \mathbf{n}_{k}
$$

Based on the transmit and receive beamforming matrices sum rate can be calculated as follows.

$$
S R=\sum_{k=1}^{K} \log _{2}\left|\mathbf{I}_{k}+\frac{\mathbf{D}_{k} \mathbf{H}_{k k} \mathbf{T}_{k} \mathbf{T}_{k}^{H} \mathbf{H}_{k k}^{H} \mathbf{D}_{k}^{H}}{\mathbf{D}_{k}\left(\sum_{j=1, j \neq k}^{K} \mathbf{H}_{k j} \mathbf{T}_{j} \mathbf{T}_{j}^{H} \mathbf{H}_{k j}^{H}+\sigma^{2} \mathbf{I}\right) \mathbf{D}_{k}^{H}}\right|
$$

The main objective is to find the best stream allocation scheme over the transmitter and receiver pairs that increases the total sum rate $(S R)$ of the network which is expressed as follows.

$$
\left\{\left(\mathbf{T}_{k}^{*}, \mathbf{D}_{k}^{*}\right)\right\}_{k \in[1, \ldots, K]}=\underset{\mathbf{T}_{k}, \mathbf{D}_{k}}{\operatorname{argmax}} S R
$$

\section{The Proposed Hierarchical Successive STREAM SELECTION}

In this study, hierarchical selection of the strongest streams with a contribution to the sum rate is proposed by performing orthogonal projections to the null space of the selected stream incrementally. The key point of this approach is to determine the stream combinations that give the highest sum rate among all the stream combination paths initialized by the pico streams. In the following sections, both stream selection and interference mitigation procedures are explained in detail. 


\section{A. Stream Selection Procedure}

Before starting the stream selection procedure, streams are identified using the singular values which are computed by applying singular value decomposition (SVD) to all channels, $\mathbf{H}_{k k}=\mathbf{U}_{k} \mathbf{S}_{k} \mathbf{V}_{k}^{H}$. In the hierarchical stream selection, the aim is to construct stream paths with a number of pico cell streams. The initialization set that only includes pico user streams is denoted as $\Xi$. The reason of this initialization is that the streams of pico users are highly affected by the interference coming from the macro cells, which causes the selection of pico streams to be very unlikely. Therefore the determination of the first stream is very crucial in order to reduce the interference of the macro cell to the pico cell. This is achieved by the selection of the first stream among the pico streams, because orthogonalization procedure is carried out when a stream is selected.

After the first stream is selected from pico streams, the selection procedure continues with the maximum singular value which increases the sum rate is chosen at each iteration from the set $\Omega$. This set keeps the track of all the available streams. The iteration continues until no more streams can be selected. The selected streams are kept in a set denoted as $\Psi$. The $\Omega$ and $\Psi$ sets are updated in each iteration of the proposed algorithm, Hierarchical Successive Null Space Stream Selection (HSNSSS), as the selected stream is taken from the set $\Omega$ and put into the set $\Psi$.

The location of the pico cells are identified using as the ratio $d / R$ where $R$ is the macro cell radius and $d$ is the distance between macro and pico BSs. Since pico cells are practically deployed at cell edges of the macro cells, the distance is taken as $\Delta \leq d / R$, where $\Delta$ is a distance threshold in order to determine the cell edge zones. To take into account this fact $\Xi$ includes only the streams of pico cell where $\Xi$ is the initial stream set.

After the stream selection, a virtual channel is defined by the precoders and decoders obtained from SVD procedure to determine Virtual Receiving Channels (VRC) and Virtual Transmitting Channels (VTC). These can be mathematically expressed as follows.

- $\quad$ Virtual Receiving Channel [7]: This channel represents the channel of the user $k^{*}$ that is selected and seen from the transmitters side.

$$
\mathbf{V R C}_{k^{*}}=\mathbf{D H}_{k^{*} k^{*}}
$$

where $\mathbf{D}$ is transpose conjugate of the $l^{*}$ column of matrix $\mathbf{U}_{k^{*}},\left(\mathbf{D}=\mathbf{U}_{k^{*}}^{l^{*} H}\right)$ and the size of this channel is $1 \times N_{T_{k}}$.

- Virtual Transmitting Channel [7]: This channel represents the channel of the user $k^{*}$ that is selected and seen from the receivers side.

$$
\mathbf{V T C}_{k^{*}}=\mathbf{H}_{k^{*} k^{*}} \mathbf{T}
$$

where $\mathbf{T}$ is the $l^{*}$ column of matrix $\mathbf{V}_{k^{*}},\left(\mathbf{T}=\mathbf{V}_{k^{*}}^{l^{*}}\right)$ and the size of this channel is $N_{R_{k}} \times 1$.

\section{B. Interference Mitigation Procedure}

After the virtual channels related to the best stream of user $k$ are obtained, the impact of this stream to the remaining streams is reduced by projecting the space spanned by the remaining potential beamformers of user $j \neq k$ orthogonally to the selected stream belonging to user $k$. The projected matrices $\mathbf{H}_{j j}^{\perp}, \forall j \neq k$ with vectors in the null space of all previously selected streams. The projection procedure is implemented in two steps [7]: In the first step the interference coming from the remaining streams to the selected stream is reduced by projecting the channel matrices $\mathbf{H}_{k k}^{\perp}$ generated at each iteration $i$ orthogonally to the VRC, $\mathbf{U}_{k^{*}}^{l^{*} H} \mathbf{H}_{k^{*} k}$, and it is calculated as

$$
\left\{\mathbf{H}_{k k}^{\perp}\right\}_{i}=\left\{\mathbf{H}_{k k}^{\perp}\right\}_{i} \mathbf{P}_{\mathbf{U}_{k^{*}}^{l * H} \mathbf{H}_{k^{*} k}}^{\perp}
$$

where $\mathbf{P}_{\left(\mathbf{U}_{k^{*}}^{l^{*} H} \mathbf{H}_{k^{*} k}\right)}^{\perp}$ is the orthogonal projection matrix parallel to matrix $\mathbf{U}_{k^{*}}^{l^{*} H} \mathbf{H}_{k^{*} k}$ and can be mathematically expressed as

$$
\mathbf{P}_{\left(\mathbf{U}^{l^{*} H} \mathbf{H}_{k^{*} k}\right)}^{\perp}=\mathbf{I}-\frac{\left(\mathbf{U}_{k^{*}}^{l^{*} H} \mathbf{H}_{k^{*} k}\right)^{H}\left(\mathbf{U}_{k^{*}}^{l^{*} H} \mathbf{H}_{k^{*} k}\right)}{\left\|\left(\mathbf{U}_{k^{*}}^{l^{*} H} \mathbf{H}_{k^{*} k}\right)\right\|^{2}} .
$$

The second step of the projection procedure is to reduce the interference generated to the remaining streams and consists in projecting the channel matrices $\mathbf{H}_{k k}^{\perp}$ generated to the VTC, $\mathbf{H}_{k k^{*}} \mathbf{V}_{k^{*}}^{l^{*}}$, and it is calculated as

$$
\left\{\mathbf{H}_{k k}^{\perp}\right\}_{i+1}=\mathbf{P}_{\mathbf{H}_{k k^{*}} \mathbf{V}_{k^{*}}^{l^{*}}}^{\perp}\left\{\mathbf{H}_{k k}^{\perp}\right\}_{i}
$$

where $\mathbf{P}_{\left(\mathbf{H}_{k k^{*}} \mathbf{V}_{k^{*}}^{l^{*}}\right)}^{\perp}$ is the orthogonal projection matrix parallel to matrix $\mathbf{H}_{k k^{*}} \mathbf{V}_{k^{*}}^{l^{*}}$ and can be similarly calculated as in the Equation 8.

In order to share the power of the corresponding cell type among the selected streams, a correction coefficient, $\alpha_{k}^{i}$, is defined for the channel matrices of each user. It can be expressed mathematically as follows.

$$
\alpha_{k}^{i}=P_{k} / q_{k}^{i}
$$

where $q_{k}^{i}$ is the number of allocated streams at iteration $i$ for user $k$ and $P_{k}$ is the transmit power of the BS $k$.

The whole procedure is described in Algorithm 1.

\section{Performance Results}

The performance of the proposed algorithm is evaluated in a heterogeneous network illustrated in Figure 3. There are one pico BS with 2 transmit antennas and one macro BS with 4 transmit antennas and each BS serves only one user equipped with 2 receive antennas. To obtain the results, channels are generated with independent and identically distributed $\mathcal{C N}(0,1)$ entries.

In the simulation, users are randomly deployed in the coverage of their own BSs. In order to observe the best location of the pico BS with respect to the macro BS, the pico BS is 


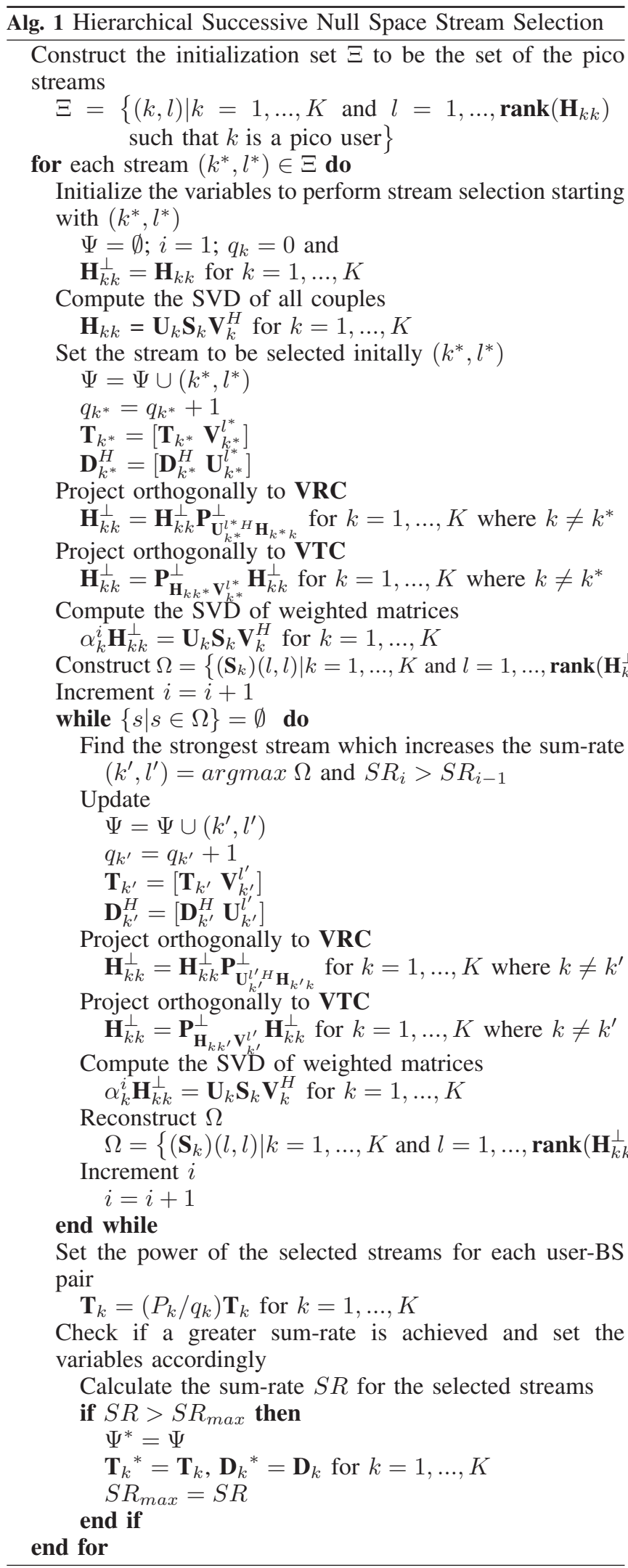

initially placed at $(500,0)$ and shifted with the pico user along the $X$-axis towards the cell edge area. The macro BS is always located at $(0,0)$.

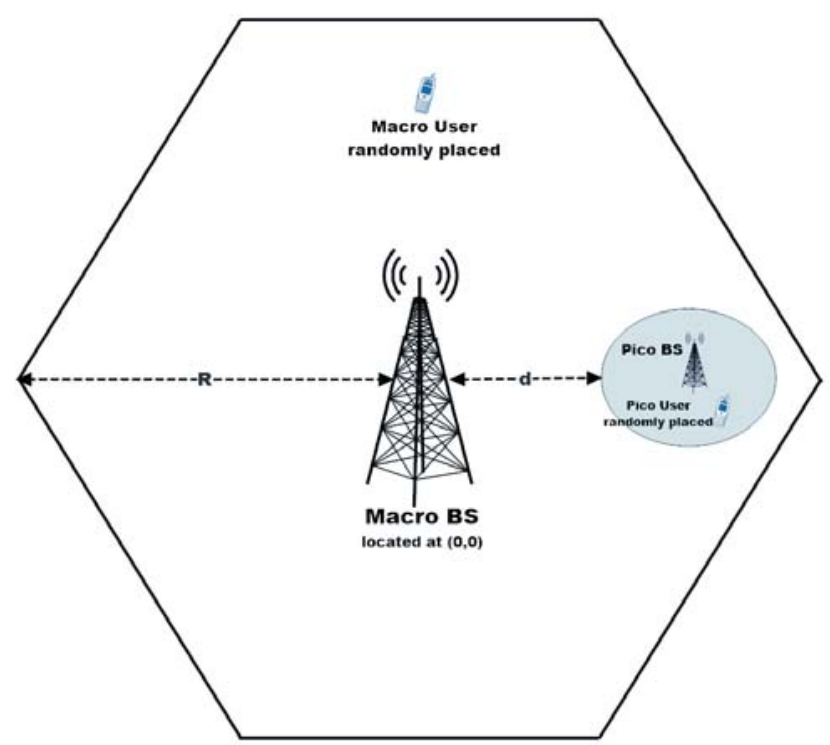

Fig. 3. Simulation Scenario for Heterogeneous Network

System parameters performed in the simulations are listed in Table I.

TABLE I. SYSTEM PARAMETERS

\begin{tabular}{|c|c|}
\hline Parameter Name & Parameter Value \\
\hline \hline Macro Cell Transmit Power & $43 \mathrm{dBm}$ \\
\hline Pico Cell Transmit Power & $30 \mathrm{dBm}$ \\
\hline Bandwidth & $10 \mathrm{MHz}$ \\
\hline Carrier Frequency & $2.1 \mathrm{GHz}$ \\
\hline Noise Power & $-174 \mathrm{dBm} / \mathrm{Hz}$ \\
\hline Macro Cell Radius & $1000 \mathrm{~m}$ \\
\hline Pico Cell Radius & $100 \mathrm{~m}$ \\
\hline Path loss (for macro) & $L_{p}=128.1+37.6 \log 10(R(\mathrm{~km})) \mathrm{dB}$ \\
\hline Path loss (for pico) & $L_{p}=140.7+36.7 \log 10(R(\mathrm{~km})) \mathrm{dB}$ \\
\hline
\end{tabular}

In order to analyze the behavior of the stream selection algorithms depending on the position of the pico cell, the order of the selected streams for each $d / R$ with their selection probabilities are shown in Table II. These results are obtained by the exhaustive search that searches all possible stream combination paths and determines the stream path with the highest performance. Since it is a brute force method, it is a very complex technique where the number of paths in this searching process can be calculated as follows.

$$
\sum_{n=1}^{N}\left(\frac{N !}{(N-n) !}\right)
$$

Considering the fact that the pico cells are practically deployed at cell edges of the macro cells, the distance beyond $\Delta=0.5$ is evaluated in this study. Therefore the streams of the pico cells are initially selected in order to reduce the interference coming from the macro cell to the pico cell. Determining the first streams from pico streams is also supported by the stream distributions given in the Table II. It can be seen that the probability of selecting the first stream 
TABLE II. STREAM PROBABILITIES ACCORDING TO THEIR ORDERS FOR $\Delta \geq 0.5$

\begin{tabular}{|c|c|c|c|c|c|}
\hline & & Strea & Orde & & \\
\hline $\mathrm{d} / \mathbf{R}$ & Stream & 1 & 2 & 3 & 4 \\
\hline \multirow{4}{*}{0.5} & Macro-1 & 0.06 & 0.188 & 0.218 & 0.446 \\
\hline & Macro-2 & 0.007 & 0.496 & 0.141 & 0.024 \\
\hline & Pico-1 & 0.365 & 0.148 & 0.432 & 0.04 \\
\hline & Pico-2 & 0.568 & 0.168 & 0.019 & 0.096 \\
\hline \multirow{4}{*}{0.6} & Macro-1 & 0.059 & 0.172 & 0256 & 0.459 \\
\hline & Macro-2 & 0.01 & 0.529 & 0.143 & 0.054 \\
\hline & Pico-1 & 0.361 & 0.148 & 0.443 & 0.043 \\
\hline & Pico-2 & 0.57 & 0.151 & 0.034 & 0.129 \\
\hline \multirow{4}{*}{0.7} & Macro-1 & 0.062 & 0.155 & 0.3 & 0.453 \\
\hline & Macro-2 & 0.013 & 0.557 & 0.141 & 0.085 \\
\hline & Pico-1 & 0.338 & 0.163 & 0.44 & 0.059 \\
\hline & Pico-2 & 0.587 & 0.125 & 0.039 & 0.173 \\
\hline \multirow{4}{*}{0.8} & Macro-1 & 0.08 & 0.135 & 0.319 & 0.449 \\
\hline & Macro-2 & 0.013 & 0.561 & 0.164 & 0.102 \\
\hline & Pico-1 & 0.332 & 0.175 & 0.433 & 0.06 \\
\hline & Pico-2 & 0.575 & 0.129 & 0.037 & 0.2 \\
\hline \multirow{4}{*}{0.9} & Macro-1 & 0.077 & 0.123 & 0.354 & 0.437 \\
\hline & Macro-2 & 0.02 & 0.59 & 0.153 & 0.118 \\
\hline & Pico-1 & 0.346 & 0.168 & 0.417 & 0.069 \\
\hline & Pico-2 & 0.557 & 0.119 & 0.047 & 0.24 \\
\hline \multirow{4}{*}{1} & Macro-1 & 0.092 & 0.13 & 038 & 039 \\
\hline & Macro-2 & 0.017 & 0.59 & 0.164 & 0.126 \\
\hline & Pico-1 & 0.367 & 0.158 & 0.385 & 0.09 \\
\hline & Pico-2 & 0.524 & 0.122 & 0.053 & 0.275 \\
\hline
\end{tabular}

from the pico user is greater than selecting it from the macro user. For instance, the first stream of the pico user, Pico- 1 is selected as the first stream with a probability of $\approx 0.35$ and the second stream of the pico user, Pico- 2 with a probability of $\approx 0.55$. These stream probabilities also show that the proposed approach provides an appropriate solution for the heterogeneous networks.

Since there are two pico streams in this scenario, Algorithm 1 constructs two stream paths by initializing the first streams by these pico streams. The obtained stream paths are compared in terms of their sum rates and the path with the highest sum rate is selected.

The performance results of the proposed algorithm is compared to the successive null space stream selection (SNSSS) [7] and the exhaustive search. In addition, the performance of the one path version of the HSNSSS algorithm is investigated with different stream initializations, such as $1 s t$ and $2 n d$ streams of pico user. The performance results can be seen in Figure 4. It can be observed that the sum rate is $1 \mathrm{bps} / \mathrm{Hz}$ improved while getting closer to the upper bound when compared to SNSSS approach. Also average user rates are increased by the HSNSSS algorithm according to the SNSSS algorithm and it is shown in Figure 5.

Besides the given results, HSNSSS approach is less com- plex compared to the exhaustive search since the number of initialization streams depends on the number of pico cells. Therefore the number of stream paths searched in HSNSSS algorithm is less than the number of all possible streams.

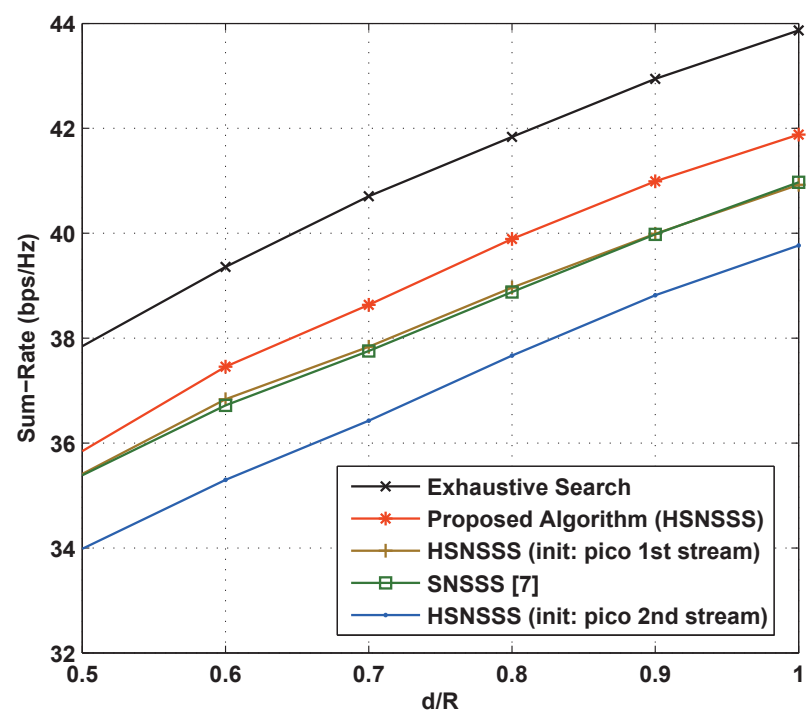

Fig. 4. Average sum rate vs. distance ratio $d / R$

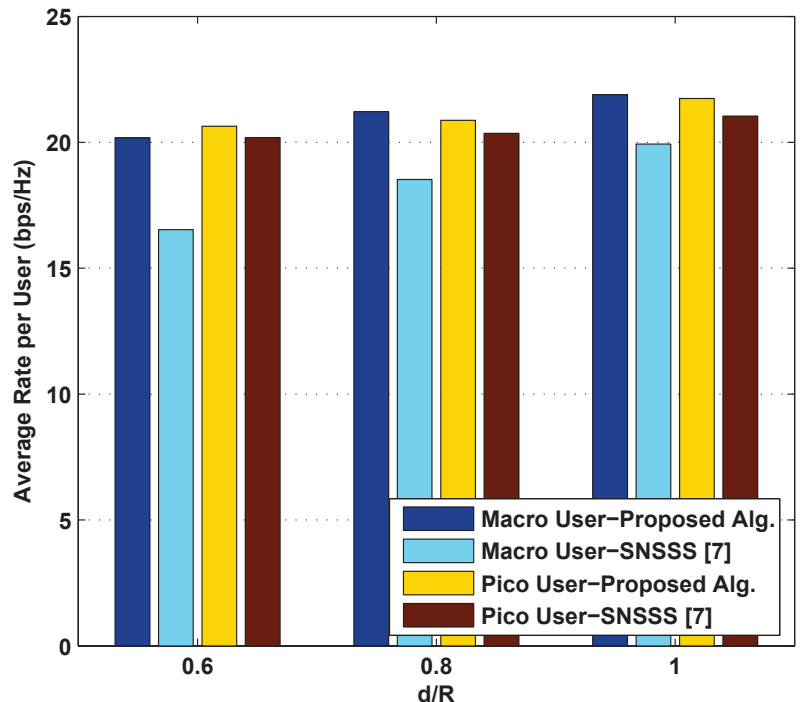

Fig. 5. Average user rates vs. distance ratio $d / R$

The comparison of the number of selected streams for each user for different distance ratios can be seen in Table III. The results confirm that the proposed method allocates more streams to users while ensuring better service to the users and increasing the sum rate. 
TABLE III. AVERAGE NUMBER OF USER STREAMS VS. DISTANCE RATIO $d / R$

\begin{tabular}{|l|l|l|l|}
\hline & $\mathrm{d} / \mathrm{R}=0.5$ & $\mathrm{~d} / \mathrm{R}=0.7$ & $\mathrm{~d} / \mathrm{R}=1$ \\
\hline \hline Macro User (Proposed Alg.) & 1.3 & 1.55 & 1.72 \\
\hline Macro User (SNSSS [7]) & 1.21 & 1.43 & 1.66 \\
\hline \hline Pico User (Proposed Alg.) & 1.82 & 1.90 & 1.98 \\
\hline Pico User (SNSSS [7]) & 1.72 & 1.87 & 1.95 \\
\hline
\end{tabular}

\section{CONCLUSiON}

In this paper, we have presented a hierarchical stream selection method for heterogeneous networks in order to deal with the interference among different cell types. The proposed algorithm selects the initial streams from the pico cells and continues to the selection procedure incrementally choosing the stream with the highest singular value if the sum rate increases with this selection. After each stream is selected, orthogonal projections are performed in order to handle the interference to and from the selected stream. The performance of the algorithm has been evaluated in a realistic simulation environment by varying the positions of pico BSs at the cell edge zone of the macro cell. The performance results indicates that the proposed hierarchical stream selection algorithm improves the sum rate and increases the number of selected streams. For future studies, additional criteria related with the stream selection process, such as interference to noise ratio (INR), can be considered in order to improve the hierarchical stream selection.

\section{REFERENCES}

[1] V. R. Cadambe and S. A. Jafar, "Interference alignment and degrees of freedom of the k-user interference channel," IEEE Transactions on Information Theory, vol. 54, no. 8, pp. 3425-3441, 2008.

[2] T. Gou and S. A. Jafar, "Degrees of freedom of the k user $m \times n$ mimo interference channel," IEEE Transactions on Information Theory, vol. 56, no. 12, pp. 6040-6057, 2010.

[3] C. Yetis, T. Gou, S. Jafar, and A. Kayran, "On feasibility of interference alignment in mimo interference networks," Signal Processing, IEEE Transactions on, vol. 58, no. 9, pp. 4771-4782, 2010.

[4] C. Suh, M. Ho, and D. N. C. Tse, "Downlink interference alignment," IEEE Transactions on Communications, vol. 59, no. 9, pp. 2616-2626, 2011.

[5] J. Schreck and G. Wunder, "Distributed interference alignment in cellular systems: Analysis and algorithms," Sustainable Wireless Technologies (European Wireless), pp. 1-8, 2011.

[6] K. S. Gomadam, V. R. Cadambe, and S. A. Jafar, "A distributed numerical approach to interference alignment and applications to wireless interference networks," IEEE Transactions on Information Theory, vol. 57, no. 6, pp. 3309-3322, 2011.

[7] M. Amara, M. Pischella, and D. Le Ruyet, "Enhanced stream selection for sum-rate maximization on the interference channel," Wireless Communication Systems (ISWCS), 2012 International Symposium on, pp. 151-155, 2012.

[8] H. Lv, T. Liu, X. Hou, and C. Yang, "Adaptive interference alignment for femtocell networks," Signal Processing (ICSP), 2010 IEEE 10th International Conference on, pp. 1654-1657, 2010.

[9] B. Guler and A. Yener, "Interference alignment for cooperative mimo femtocell networks," IEEE Global Communications Conference (GLOBECOM), 2011 IEEE, pp. 1-5, 2011.

[10] W. Shin, W. Noh, K. Jang, and H.-H. Choi, "Hierarchical interference alignment for downlink heterogeneous networks," Wireless Communications, IEEE Transactions on, vol. 11, no. 12, pp. 4549-4559, 2012. 